\title{
Severe Left Main Coronary Stenosis Due to Aortic Bioprosthesis Implantation:
} A Case Report

\author{
Huimin Cui, PhD*, Shixiong Wei, MD*, Lin Zhang, PhD, Shengli Jiang, PhD \\ Department of Cardiovascular Surgery, Chinese PLA General Hospital, Beijing, China
}

\section{ABSTRACT}

Coronary artery ostial stenosis is a common but lifethreatening complication that usually presents right after valve implantation, especially in transcatheter aortic valve implantation (TAVI) procedure. However, as reported in our case, it may also have a late delayed presentation in valve replacement through median sternotomy. Here, we present a rare case of one patient who underwent percutaneous coronary intervention (PCI) for severe stenosis of the left main (LM) coronary artery six months after Mosaic aortic bioprosthesis implantation.

\section{INTRODUCTION}

The excellent hemodynamics and improvement of durability of currently available third-generation bioprosthetic aortic valves have reduced the incidence of bioprosthetic valve dysfunction [Hirota 2016]. However, non-structural valve dysfunction still is seen in clinical practice, among which coronary artery ostial stenosis is a rare but life-threatening complication [Funada 2006]. The frequency of this serious event is reported to be $0.7 \% \sim 3.5 \%$ in transcatheter aortic valve implantation (TAVI) procedure [Dvir 2012], whereas it is $1 \% \sim 5 \%$ in the subacute phase following surgical aortic valve replacement (AVR) [Funada 2006]. Here, we present a case of a 66-year-old woman who underwent percutaneous coronary intervention (PCI) for severe left main (LM) stenosis six months after Mosaic aortic bioprosthesis implantation.

\section{CASE PRESENTATION}

A 66-year-old woman, who received surgical AVR for a severe bicuspid aortic stenosis six months ago, was readmitted to our department for paroxysmal left shoulder pain. A $21 \mathrm{~mm}$ Mosaic aortic bioprosthesis was used in the previous surgery, and the pledgets were placed under the natural annulus, which sized $22 \mathrm{~mm}$. The ostia of coronary arteries,

Received fune 17, 2020; accepted fuly 28, 2020.

*Both authors contributed equally to this work.

Correspondence: Shengli fiang, Department of Cardiovascular Surgery, Chinese PLA General Hospital, 28 Fuxing Road, Beijing, 100853, China; +86-13701014395; fax +86-10-68299399 (e-mail: jiangsl301@sina.com).
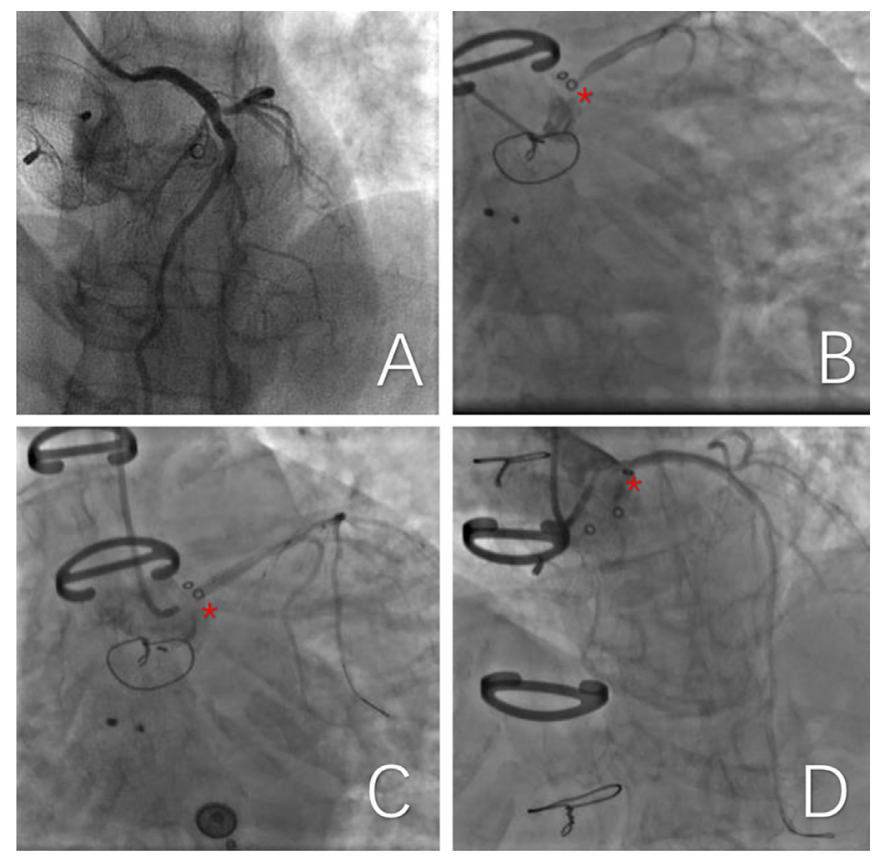

Figure 1. A, LM was normal before bioprosthesis implantation. B, An $85 \%$ tubular stenosis of the proximal LM. C, A stent was released at the proximal LM. D, Angiography showed no dissection. LM: left main coronary artery; *indicates radiopaque stent post eyelet in aortic protrusion in Mosaic
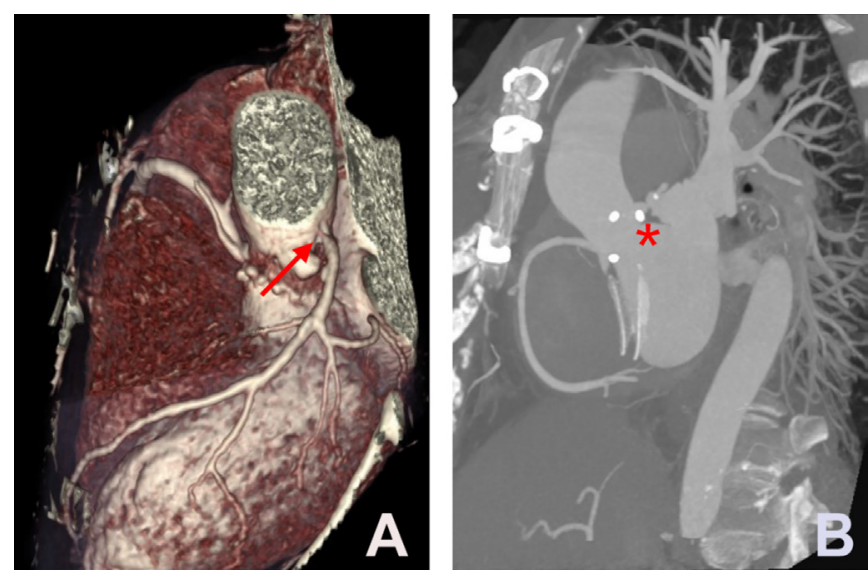

Figure 2. CTA showed extreme stenosis of the LM (arrow). *indicates radiopaque stent post eyelet in aortic protrusion in Mosaic. CTA: computer tomography angiography; LM: left main coronary artery 
which were revealed to be normal by preoperative angiography (Figure 1A), were well exposed under direct vision after tying sutures.

The computer tomography angiography (CTA) of coronary artery, which was suggested by electrocardiogram monitoring with an abnormal ST segment changes of severe myocardial ischemia, found an unexpected severe stenosis of the LM (Figure 2). PCI treatment immediately was given, and intraoperative angiography revealed an $85 \%$ tubular stenosis in the proximal LM (Figure 1B). Intravascular ultrasound (IVUS) revealed fibrous plaque and hyperechoic image changes in the proximal LM with the narrowest area measuring $3.34 \mathrm{~mm}$. Finally, a $3.5 \times 18 \mathrm{~mm}$ cobalt based alloy rapamycin eluting stent was delivered to the proximal LM and released at $12 \mathrm{~atm}$ after a series of successful percutaneous transluminal coronary angioplasty (PTCA) treatment (Figure 1C). Angiography showed no dissection, thrombolysis in myocardial infarction (TIMI) grade 3 (Figure 1D). The patient was discharged uneventfully three days later.

\section{DISCUSSION}

Coronary ostial stenosis is a rare but fatal complication of AVR, which differs from that of arteriosclerosis. It usually is diagnosed by angiography and treated with coronary artery bypass grafting. The cause of this complication generally is recognized as an intimal thickening and fibrous proliferation, however, the detailed mechanism has not yet been fully demonstrated [Funada 2006]. Acute traumatic tissue damage and tissue remodeling during the trauma healing process for direct cannulation of the coronary artery ostium and myocardial perfusion, a reaction to turbulent flow around the prosthetic valves, and an immunological reaction to the heterograft are considered to be possible pathophysiologic mechanisms for its formation [Funada 2006; Tukiji 2004]. Tatsuishi et al suggested coronary artery orifices located near a commissure and distance between the coronary artery and commissure $\leq 3 \mathrm{~mm}$ are at risk of coronary flow obstruction by the stent post of the bioprosthetic valve [Tatsuishi 2015].

The pathogenesis of coronary ostial stenosis in the present case is similar to those previously reported [Funada 2006;
Tukiji 2004]. However, inappropriate location of the bioprosthesis may also contribute to its occurrence, of which one aortic protrusion is located against the aortic sinus wall causing compression or distortion of the LM with the beating heart, whereas the ostia is not affected. Nevertheless, without direct-vision confirmation, all possible causes do exist.

The late delayed occurrence of coronary obstruction renders this complication, already burdened by an extremely high mortality, even more difficult to manage [Buscaglia 2018]. Therefore, an adequate and careful assessment of anatomic features and the bioprosthesis is of paramount importance. Even though rare, some complications are sudden and fatal [Buscaglia 2018]. Since bioprostheses now are increasingly preferred to mechanical valves in the aortic position, the best way to limit unsuccessfulness or complications is by exquisite attention to detail at all phases of the operation.

\section{REFERENCES}

Buscaglia A, Tini G, Bezante GP, et al. 2018. Sudden death after valvein-valve procedure due to delayed coronary obstruction: a case report. Journal of Medical Case Reports 12:247-252.

Dvir D, Webb J, Brecker S, et al. 2012. Transcatheter aortic valve replacement for degenerative bioprosthetic surgical valves: results from the global valve-in-valve registry. Circulation. 126:2335-44.

Funada A, Mizuno S, Ohsato K, et al. 2006. Three Cases of Iatrogenic Coronary Ostial Stenosis After Aortic Valve Replacement. Circ J 70:1312-1317.

Hirota M, Isomura T, Yoshida M, et al. 2016. Subvalvular Pannus Overgrowth after Mosaic Bioprosthesis Implantation in the Aortic Position. Ann Thorac Cardiovasc Surg 22:108-111.

Tatsuishi W, Nakano K, Kubota S, et al. 2015. Identification of Coronary Artery Orifice to Prevent Coronary Complications in Bioprosthetic and Transcatheter Aortic Valve Replacement. Circulation Journal 79:2157-61.

Tukiji M, Akasaka T, Wada N, et al. 2004. Bilateral coronary ostial stenosis after aortic valve replacement with Freestyle stentless bioprosthesis: A case report. J Cardiol 44: 207-213. 